\title{
Design and Simulation of Smart Communication System for Unmanned Arial Vehicles
}

\author{
Prashant Mani ${ }^{1}$, Pankaj Singh ${ }^{2}$, Abhishek Singhal ${ }^{3}$, Apoorv Katiyar ${ }^{4}$ \\ 1,2,3,4 SRM Institute of Science and Technology, NCR Campus, India
}

\begin{abstract}
In recent years, the use of drones has drastically increased as the evolution of drone use in commercial sectors and reduced costs of the hardware. Earlier drone services were mostly used for military operations but nowadays the Unmanned Arial Vehicles (UAV) system is very advanced and its applications are not limited to military operations. The recent years have also witnessed a network evolution of UAVs from single ground to air network to multi-UAV network systems along with usage of wireless public networks like LTE which can act as UAV communication channel. In the proposed project, a communication system used in the UAS system is simulated to analyze the UAV behavior under different conditions with respect to mission planning and the communication networks used. A comprehensive study is done on communication networks used in controlling UAVs. For a safer approach, the proposed model is simulated using available software instead of hardware implementations. ArduPilot SITL, MAVProxy and Mission Planner are used to simulate the UAV system virtually. Whereas network simulations of Wi-Fi and LTE network are done with the help of NS-3 on a separate platform. Various network parameters like network delay, throughput, etc., are graphically represented.
\end{abstract}

Keywords - Communication system, NS-3, SITL, UAV simulation.

\section{INTRODUCTION}

$\mathrm{U}$ $\mathrm{AV}_{\mathrm{s}}$ or unmanned aerial vehicles are part of the unmanned aerial system [1]. UAVs are pilotless aircraft [2] or aerial vehicles that can be remotely operated. An unmanned aerial vehicle consists of sets of sensors and electronic devices that made them capable of flying without the need of an actual pilot. UAVs are part of the aerial vehicles system for a very long time.UAVs can be operated in both guided and auto mode i.e., the operator can manually control the vehicle at every instant of time or UAVs can fly autonomous missions with the help of preloaded commands on the onboard chip of the UAV. Earlier, UAVs were designed for carrying out military and other secret operations. The fact that differentiates a UAV from missiles is that UAVs are recoverable and are capable of performing airstrikes at the same time. UAVs were used as recoverable aerial weapons in the later stages of development. The concept of aerial photography with the help of UAVs was proved to be a major breakthrough in aspects of war. Although many countries and organizations were involved in the development and use of UAVs, it can be said that U.S.A and Israel have contributed the most to the development of UAVs. Recently, with the commercialization of drones or UAVs, the use of these aerial machines is proposed in sectors of agriculture, health services, packaging services etc[3][4].

Apart from UAV as a unit, a control center or ground control station can also be considered as part of an unmanned aerial system. Ground control stations are used as remote controllers with the integration of various other hardware modules for analyzing the vehicle dynamics and performance during operations. Communication system plays a vital role in the unmanned aerial system. A robust communication system is required for controlling UAVs in the air. Generally, UAVs or drones are controlled by radio controllers over a frequency of 2.4 and $5.8 \mathrm{GHz}$, where one frequency link is used for sending commands to the drone and the other link is utilized for receiving the telemetry and payload data from the UAV[5]. As the communication is wireless so security threats are always involved. Spread-spectrum modulation techniques are used to maintain the confidentiality of data transmitted or received over the network. Frequency-hopping spread spectrum and direct sequence spread spectrum [6] are used to transmit the data over the communications network. Thus, the use of these modulation techniques is the first line of defense for a secure UAV communication system. OFDM or orthogonal frequencydivision multiplexing techniques are also used for faster travel rates of payload data. Nowadays, the UAVs can also be operated over wireless public networks like Wi-Fi, 4G LTE[7], etc., which increases the security threat to the UAV communication system. Several techniques and encryption algorithms [8][9][10][11]are suggested and used to avoid security breaches in the network. 


\section{LITERATURE REVIEW}

For a comprehensive study, the literature survey is broadly disintegrated into four parts. A literature survey on brief history is done in the first part. In the second part, a discussion is made on the classification of UAVs. In the third part, a comprehensive study is done on the architecture of the UAV communication system and its vulnerabilities of the system. In the last part, the survey is made on the studies related to UAV and network simulation models.

\section{A. History of UAVs}

The drones and UAVs were earlier introduced in World War I [12] and their use was confined to military operations [13]. Although the concepts and designs related to UAVs were introduced years ago. The first UAV designs were introduced in the age of Pythagoras [14]. In the year 1843, Leonardo the Vinci came up with a flying machine [15]. Several such concepts were introduced which were powered by steam and water vapors. It was in the year 1849 when drones were deployed as combat aerial vehicles. In the year 1896, a camera was mounted on a rocket by Alfred Nobel which also induced a concept of aerial photography by flying machines. In the year 1900, Nikola tesla[16] came up with the idea of controlling air balloons which was a modified concept of the radio-controlled boat introduced by tesla in 1898. During World War I the U.S. Army was working on aerial torpedoes which were recognized as the Kettering bug model[17]. After World War, more nations and organizations got involved in using drone models. This started the evolution of UAVs where the U.S. introduced Curtiss N2C-2, which is also considered as first UAV. Later on, contributions were made by Israel and after that U.S. and Israel worked on several drone technologies. Along the time with redesigning and modifications of UAVs, it was between 2005-2010 [18] when these aerial machines having quadrotor mechanisms were introduced. This gave rise to the commercialization of UAVs in sectors of health, surveying, agriculture, etc [19].

\section{B. Classification of UAVs}

UAVs can broadly be differentiated into fixed-wing UAVs, Multi-rotor UAVs and balloons or large UAVs [20]. UAVs can also be differentiated based on their payload carrying capacity., fuel capacity, endurance, range, wing types, take-off trajectories, etc [21][22][23].

\section{Architecture of UAVs}

The architecture of the UAV communication system [24] involves transmitters and receivers on ground control stations and UAVs. There are several methods to control a UAV which include radio channel, satellite and wireless public network communication. Nowadays, multi-UAV systems are used which communicate with each other with the help of network topologies. Several modulation techniques are also involved to provide a secure communication system.

\section{Simulation of drone technologies}

Nowadays a safer and smarter approach is adopted by researchers which includes simulation of technologies before deploying the actual models. Studies related to testing of a simulated quadcopter [25][26] or a multi-UAV network are performed for understanding the problem and possible failures. Research is also done on the communication network and the protocols like MAVLink [27] which are associated with a UAV communication system. An inspection is done on the vulnerability [28] of the MAVLink protocol and several encryption techniques [29][30] and methods are suggested to deploy a secure communication system of UAVs. Similarly, various network simulators [31][32] are used for research in UAV communication systems.

\section{TERMINOLOGY}

Some important definitions need to be understood for a better understanding of concepts that were kept in mind at the time of research work. Starting with drone lingual, arming a drone is referred to as providing power to the motors of the drone. The angular movement of drones with respect to the longitudinal, latitudinal and vertical axis is termed as roll, pitch and yaw respectively. Propellers are the wings of a drone that are attached to a motor to generate enough thrust for the drone to perform movements. Take-off generally refers to ascending of a drone in the air from the ground position. Networking concepts include packets, interface, ports and protocols. Packets are the most fundamental unit in networking architecture which envelops the data. Ports are the virtual or hardware connection points whereas interfaces can be defined as the communication points of a system that helps in the interaction between physical and virtual entities with or within the system. Protocols can be defined as the set of rules and regulations that determine the flow of data within a system.

\section{METHODOLOGY}

In this section, the outline of the work done in this study is presented. The process is divided into four steps namely requirement analysis, coding, testing and computation. First, we start by setting up a build environment within which the software modules are downloaded and installed. In this work virtual box is set up on which ubuntu version 18.04 is emulated. A part of this study is based on working on this Linux environment. The main software modules and firmware required for this study include SITL, Ground control station software and FlyNetSim. Most of these modules are built on $\mathrm{C}++$ and Python libraries. 


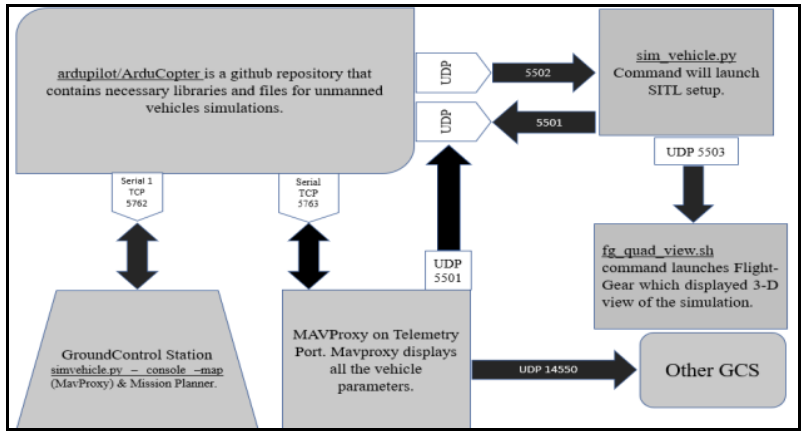

FIGURE 1 - Architecture of SITL[33]

- SITL and MAVLink - SITL[33] stands for software in the loop. SITL contains the firmware of different kind of vehicles which can be simulated. So, a quadcopter is simulated with the help of SITL in this work. Along with the vehicles, sensors like gimbals, optical flow sensors can also be simulated with the vehicle. Autopilot is supported by an open-source software suite called ArduPilot. The ardupilot firmware can be cloned from the Github repository. MAVLink is an open-source lightweight protocol specially designed for UAV communication system. MAVLink protocol has an 8-bit header file along which the data travels in the UAV communication system.

- FlyNetSim - FlyNetSim[32] is an open-source simulator that integrates a network simulator with SITL. Thus, with the help of network simulator NS-3, UAV can be simulated over Wi-Fi and LTE network. FlyNetSim uses ZMQ/czmq libraries to bind the ports between SITL and NS-3.

- Ground Control Station- These software modules are helpful in mission planning for UAVs and analyzing the telemetry data. Mission planner and Q ground control [25] are such types of software modules that help in-vehicle monitoring and controlling.

\section{RESULTS AND DISCUSSION}

The result and data analysis of this study is divided into two parts. In the first part of the study, the telemetry data is obtained from a simulated quadcopter is obtained and graphically represented with the help of a mission planner. In the second part communication networks like Wi-Fi and LTE are simulated over which the UAV is simulated and graphical representation of network data is produced.

$>$ Analysis of UAV Communication system based on telemetry data.

> Analysis of UAV communication system based on networking.

In the first part of the study, the UAV is simulated with the help of SITL, MAVProxy and Mission planner. Telemetry data received from the UAV is obtained from three test cases where a simulated operates in auto mode. The missions are planned with the help of a mission planner. In the test cases, the UAV heads to a single waypoint, circular waypoints and random waypoints as shown in figure 1.

\section{A. Single Waypoint Mission}

In this mission, the quadcopter is operated in guided mode and performs a take-off to $50 \mathrm{~m}$. Then the mode is switched to auto and the quadcopter starts to ascend to $100 \mathrm{~m}$ altitudes as specified in auto mission and then heads towards the single waypoint. As the copter reaches the waypoint, the copter descends vertically to land and the motors are disarmed.

\section{B. Circular Waypoint Mission}

This mission is planned in such a way that the copter follows circular waypoints. In this mission, the quadcopter is operated in guided mode and performs a take-off to $50 \mathrm{~m}$. Then the mode is switched to auto and the quadcopter starts to ascend to $100 \mathrm{~m}$ altitudes as specified in the auto mission and then heads along with circular waypoints. As the copter reaches the last waypoint, the copter descends vertically to land and the motors are disarmed.

\section{Random Waypoint Mission}

This mission is planned in such a way that the copter follows the random waypoint. As specific commands can also define at specific waypoints so in this the copter is commanded to loiter around a $60 \mathrm{~m}$ circle performing three rotations over the second waypoint. In this mission, the quadcopter is operated in guided mode and performs a take-off to $10 \mathrm{~m}$. Then the mode is switched to auto and the quadcopter starts to ascend to $30 \mathrm{~m}$ altitude as specified in the auto mission and then heads towards the next waypoint. As the copter reaches the last waypoint, the copter descends vertically to land and the motors are disarmed.

As the quadcopter follows these automated missions, telemetry data about vehicle dynamics and status is recorded and graphically represented with the help of the mission planner in each case as shown in figures 3,4 and 5 respectively.

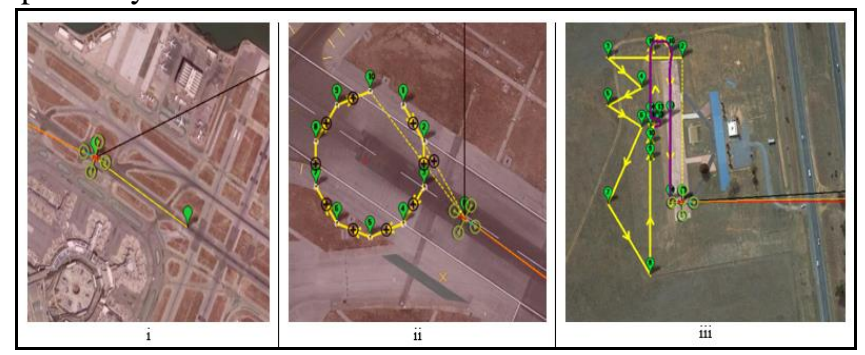

FIGURE 2 - Automated missions planned for quadcopter (i- Single waypoint; ii- Circular waypoint; iii- Random waypoint)

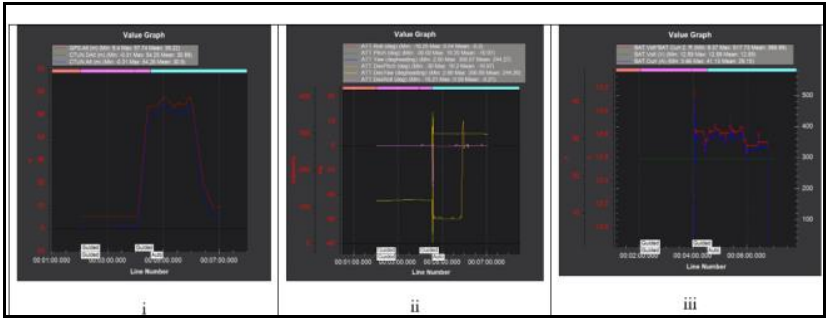

FIGURE 3 - Graphical representation of quadcopter dynamics for single waypoint mission (i- Altitude hold; ii- Pitch, roll \& raw movements; iii- Battery parameters) 


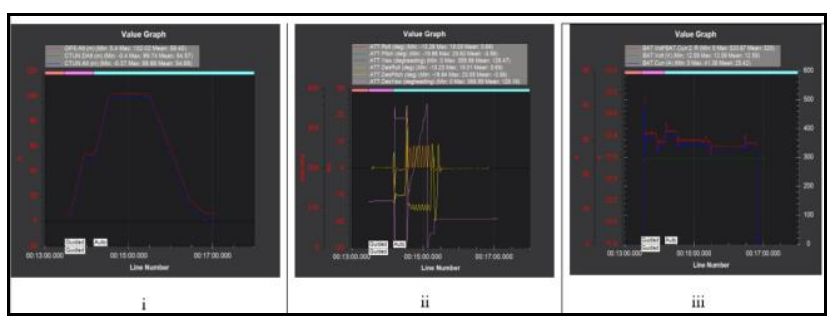

FIGURE 4 - Graphical representation of quadcopter dynamics for circular waypoints mission (i- Altitude hold; ii- Pitch, roll \& raw movements; iii- Battery parameters)

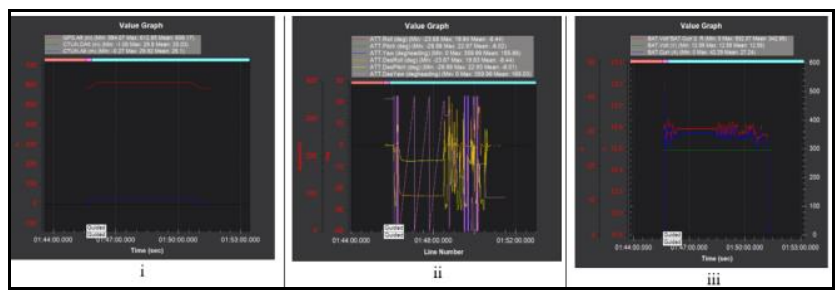

FIGURE 5 - Graphical representation of quadcopter dynamics for random waypoints mission (i- Altitude hold; ii- Pitch, roll \& raw movements; iii- Battery parameters)

Thus, after analyzing the graphs based on telemetry data, it can be said that the quadcopter follows commands in each waypoint mission with accuracy with very negligible differences in mean values which can be avoided by further calibration.

In the second part of the study, the focus is done on the simulation of different networks like Wi-Fi and LTE with the help of NS-3. The study is divided into three test cases where the quadcopter is simulated over Wi-Fi, LTE and multinetwork respectively as shown in figure 6 . In the case of a multi-network environment, the control commands are sent over a Wi-Fi network whereas telemetry commands are sent over an LTE network. For each test case, the simulated copter performs a vertical take-off for $10 \mathrm{~m}$. As the vehicle attains the height the vehicle heads for $10 \mathrm{~m}$ in forward, right, backward and left direction respectively. The output is analyzed on NS-3 compatible software modules like Wireshark as shown in figures 7, 8 and 9, NetAnim and Tracemetrics as shown in figures 10 and 11 .

Networking parameters are graphically visualized where packets sent per second, throughput, goodput, delay in TCP streams can be observed. Apart from that network delay is also realized. For communication over Wi-Fi, the network delay is 1 millisecond whereas for communication over LTE network the network delay is 4.2 milliseconds. For communication over a multi-network environment network delay is 1.2 milliseconds. The LTE network delay can be a bit inaccurate which can be corrected by adjusting the uplink and downlink speed. For each case, the output is obtained. Throughput and goodput of Wi-Fi and multi-network are displayed in table 2.

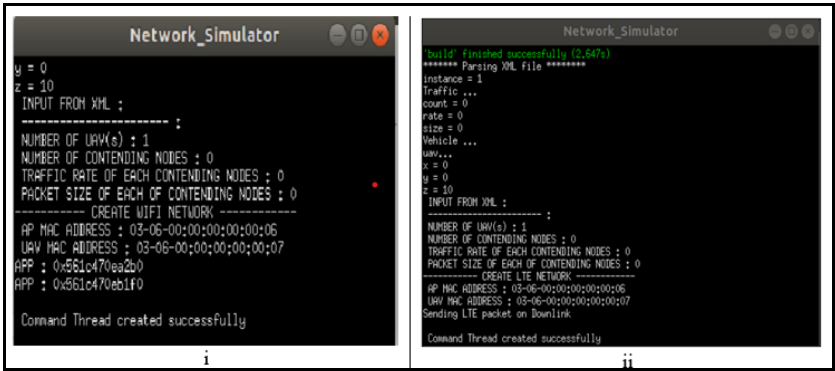

FIGURE 6 - Network simulated over NS-3 (i- Wi-Fi network; iiLTE network)

Table 1 - Throughput and Goodput of data as calculated by Tracemetrics analyser

\begin{tabular}{|c|c|c|}
\hline \multicolumn{3}{|c|}{ Communication over Wi-Fi network } \\
\hline Node & Throughput & Goodput \\
\hline 0 & 3832.8265910847717 & 3581.7572790517906 \\
\hline 4 & 4024.9076698445415 & 3818.0537662114575 \\
\hline \multicolumn{3}{|c|}{ Communication over the multi-network environment } \\
\hline Node & Throughput & Goodput \\
\hline 0 & 217.49914382673128 & 0.0 \\
\hline 4 & 6912.213700847871 & 6072.049315580278 \\
\hline
\end{tabular}

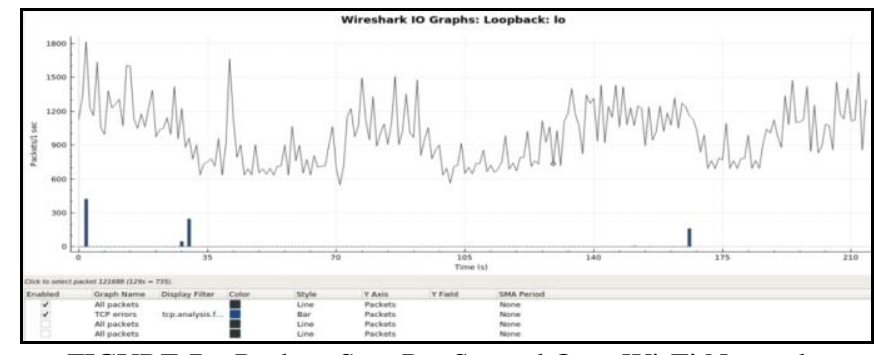

FIGURE 7 - Packets Sent Per Second Over Wi-Fi Network

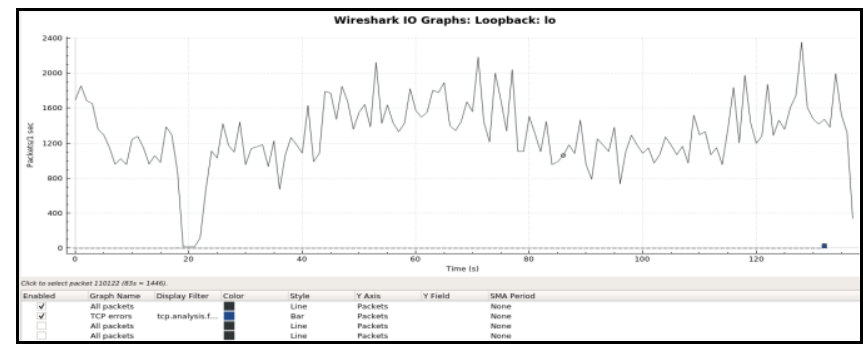

FIGURE 8 - Packets Sent Per Second Over LTE Network

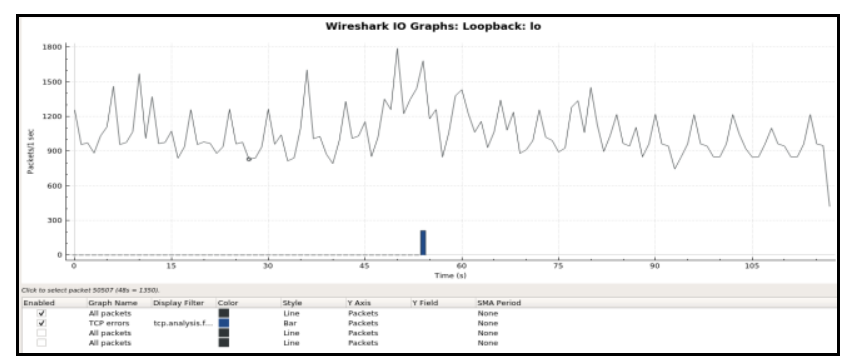

FIGURE 9 - Packets Sent Per Second Over Multi-Network Environment 


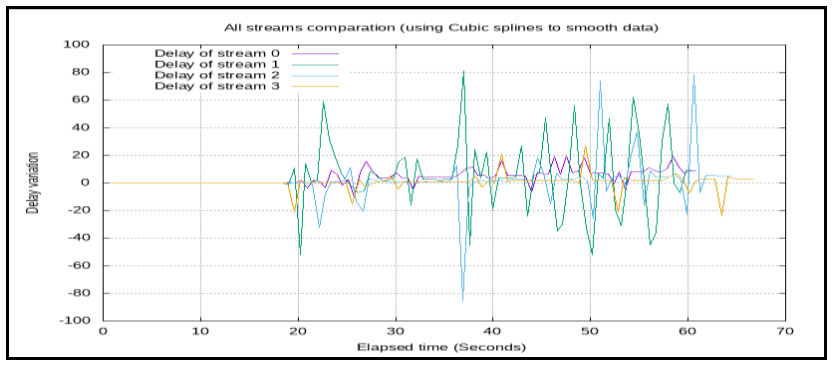

FIGURE 10 - Delay in TCP Streams Over Wi-Fi Network

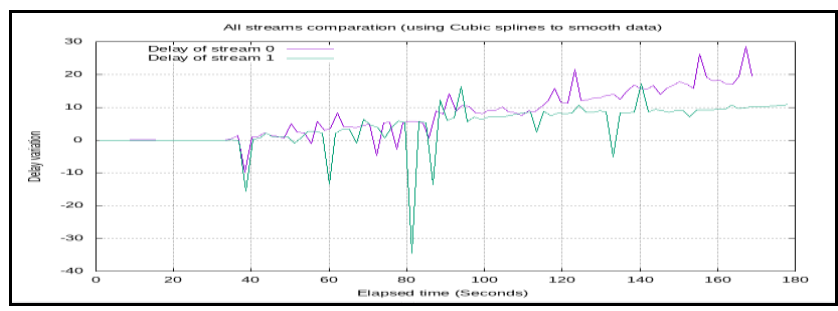

FIGURE 11-Delay in TCP Streams In Multi-Network Environment.

\section{CONCLUSION}

These days more research and development work is focused on simulations which helps in observing the behavior of the model without any hardware requirements. Vehicle simulations are extremely useful before deploying the actual models because it helps in determining the faults without the use of hardware modules which in turn saves money. The quality and efficiency of the model can also be improved with the data sets obtained by the simulation. In this work, UAV is simulated and data is obtained and analyzed based on telemetry data and network used. In, the first part of the work the telemetry data is obtained and graphically visualized by parsing the MAVLink information. The results obtained in this part show accuracy with negligible differences. In the second part of the study, communication networks are simulated which are used to control the simulated quadcopter. The results are obtained by monitoring network parameters. Therefore, in this research work, the performance analysis of UAV communication is done successfully with the help of simulations. It can be concluded that the simulation of the UAV communication system helps determine the behavior of aerial vehicles which can be implemented in realistic scenarios.

\section{LIMITATIONS AND FUTURE STUDIES}

In this study, a quadcopter is simulated with the help of SITL firmware. This firmware can also be uploaded on the onchip board of a real drone to perform guided and automated missions. Thus, updated software and firmware should be used with real drones to avoid any vehicle crash or disfunction. The possible future studies can be incorporated by modifying the dynamics of vehicle firmware in SITL for more realistic observation. More robust network simulators can be used to simulate the UAV networks for comprehensive and analytical study. The SITL should also be tested for more complex missions and networks for more accurate realistic predictions.

\section{ACKNOWLEDGMENT}

I would like to express my sincere gratitude towards my colleagues Dr. Pankaj Singh and Abhishek Singhal, Assistant professor, Dept. of Electronics and communication engineering, SRM IST, NCR Campus, India for his valuable guidance and support throughout the project, without which this work would not have been possible. I am truly grateful to him for his valuable time and encouragement.

\section{References}

[1] J. J. Hu, A. Lanzon, "An innovative tri-rotor drone and associated distributed aerial drone swarm control", Robotics and Autonomous Systems, vol. 103, (2018), pp. 162-174.

[2] "Uncrewed Aircraft Systems (UAS) | Department of Geography \& Environment." https://geog.sfsu.edu/UAS (accessed May 22, 2021).

[3] "237 Ways Drone Applications Revolutionize Business | Droneii." https://droneii.com/237-ways-droneapplications-revolutionize-business (accessed May 22, 2021).

[4] S. Hayat, E. Yanmaz, and R. Muzaffar, "Survey on Unmanned Aerial Vehicle Networks for Civil Applications: A Communications Viewpoint," IEEE Communications Surveys \& Tutorials, vol. 18, no. 4, (2016), pp. 2624-2661.

[5] D. Mototolea, "A study on the actual and upcoming drone communication systems," International Symposium on Signals, Circuits and Systems (ISSCS), (2019), pp. 1-4, July 12-15.

[6] M. Hasan, J. M. Thakur, and P. Podder, "Design and Implementation of FHSS and DSSS for Secure Data Transmission", International Journal of Signal Processing Systems, vol. 4, no. 2, (2016), pp. 144-149.

[7] F. Maiwald and A. Schulte, "5.3 - Using LTE-networks for UAS-communication," pp. 166-175, 2020, doi: 10.5162/etc2016/5.3.

[8] S. Islam, F. Ajmal, S. Ali, J. Zahid, and A. Rashdi, "Secure end-to-end communication over GSM and PSTN networks," IEEE International Conference on Electro/Information Technology, (2009), pp. 323-326, June 7-9.

[9] H. C. Nguyen, R. Amorim, J. Wigard, I. Z. Kovacs, T. B. Sorensen, and P. E. Mogensen, "How to Ensure Reliable Connectivity for Aerial Vehicles over Cellular Networks," IEEE Access, vol. 6, (2018), pp. 12304-12317.

[10] M. Faraji-Biregani and R. Fotohi, "Secure communication between UAVs using a method based on smart agents in Unmanned Aerial Vehicles," The Journal of Supercomputing, vol. 77, (2021), pp. 5076-5130.

[11]Y. Zhou et al., "Secure Communications for UAVEnabled Mobile Edge Computing Systems," IEEE Transactions on Communications, vol. 68, no. 1, (2020), pp. $376-388$. 
[12] S. Pop, A. Luchian, R.-G. Zmădu, And E. Olea, "The Evolution of Unmanned Aerial Vehicles," Review of the Air Force Academy, vol. 15, no. 3, (2017), pp. 125-132.

[13] J. F. Keane and S. S. Carr, "A brief history of early unmanned aircraft," Johns Hopkins Apl Technical Digest, vol. 32, no. 3, (2013), pp. 558-571.

[14] V. Prisacariu, "The History and the Evolution of UAVs from the Beginning Till the 70s," Journal of Defense Resources Management., vol. 8, no. 1, (2017), pp. 181189.

[15] M. E. Rosheim, Leonardo's Lost Robots. 2006.

[16] U.S. Army, "Unmanned Aircraft Systems Roadmap 20102035," Fed. Am. Sci., pp. 1-140, 2010.

[17] "History of U.S. Drones | Understanding Empire: Technology, Power, Politics." https://understandingempire.wordpress.com/2-0-a-briefhistory-of-u-s-drones/ (accessed May 22, 2021).

[18] "A Not-So-Short History of Unmanned Aerial Vehicles (UAV) - Consortiq." https://consortiq.com/short-historyunmanned-aerial-vehicles-uavs/ (accessed May 22, 2021).

[19]H. Shakhatreh, "Unmanned Aerial Vehicles (UAVs): A Survey on Civil Applications and Key Research Challenges," IEEE Access, vol. 7, (2019), pp. 4857248634.

[20] Y. Huo, X. Dong, T. Lu, W. Xu, and M. Yuen, "Distributed and multi-layer UAV network for the nextgeneration wireless communication," arXiv, vol. 1, (2018), pp. 1-13.

[21]A. G. Korchenko and O. S. Illyash, "The generalized classification of Unmanned Air Vehicles," IEEE 2nd International Conference Actual Problems of Unmanned Air Vehicles Developments Proceedings (APUAVD), (2013), pp. 28-34, September 15-17.

[22] M. Arjomandi, S. Agostino, M. Mammone, M. Nelson, and T. Zhou, "Classification of unmanned aerial vehicles," Rep. Mech. Eng. class, Univ. Adelaide, Adelaide, Aust., pp. 1-48, 2006.

[23] G. Singhal, B. Bansod, and L. Mathew, "Unmanned Aerial Vehicle Classification , Applications and Challenges: A Review," Preprint, 2018110601, (2018), pp. 1-19.

[24]L. Krichen, M. Fourati, and L. C. Fourati, "Communication architecture for unmanned aerial vehicle system", International Conference on Ad-Hoc Networks and Wireless (Part of the Lecture Notes in Computer Science book series), vol. 11104, (2018), pp. 213-225.

[25] A. I. Hentati, L. Krichen, M. Fourati, and L. C. Fourati, "Simulation Tools, Environments and Frameworks for UAV Systems Performance Analysis," 14th International Wireless Communications \& Mobile Computing Conference (IWCMC), (2018), pp. 1495-1500, June 2529.

[26] R. Vega Astorga, "Simulation of a Quadrotor Unmanned Aerial Vehicle," no. July, 2016.

[27] A. Koubaa, A. Allouch, M. Alajlan, Y. Javed, A. Belghith, and M. Khalgui, "Micro air vehicle link (MAVlink) in a nutshell: A survey," IEEE Access, vol. 7, (2019), pp. 87658-87680.
[28] J. A. Marty, "Vulnerability Analysis of the Mavlink Protocol," p. 142, 2014, [Online]. Available: http://www.dtic.mil/cgi-bin/GetTRDoc?AD=ADA598977.

[29] S. Atoev, O. J. Kwon, C. Y. Kim, S. H. Lee, Y. R. Choi, and K. R. Kwon, "The Secure UAV Communication Link Based on OTP Encryption Technique," Eleventh International Conference on Ubiquitous and Future Networks (ICUFN), (2019), pp. 1-3, July 2-5.

[30] K. Domin, E. Marin, and I. Symeonidis, "Security Analysis of the Drone Communication Protocol: Fuzzing the MAVLink protocol," 37th WIC Symposium on Information Theory, Benelux, (2016), pp. 07, May 19-20

[31] Q. Liu, H. Wang, Y. Sun, and T. Han, "A Multi-UAVs Communication Network Simulation Platform using OPNET Modeler," IEEE International Conference on Communications (ICC), (2020), pp. 1-6, June 7-11.

[32] S. Baidya, Z. Shaikh, and M. Levorato, "FlynetSim: An open source synchronized UAV network simulator based on ns-3 and ardupilot," ACM International Conference on Modelling, Analysis and Simulation of Wireless and Mobile Systems (MSWIM '18), (2018), pp. 37-45, October 28- November 2.

[33] "SITL Simulator (Software in the Loop) - Dev documentation." https://ardupilot.org/dev/docs/sitlsimulator-software-in-the-loop.html (accessed May 22, 2021).

\section{Creative Commons Attribution License 4.0 (Attribution 4.0 International, CC BY 4.0)}

This article is published under the terms of the Creative Commons Attribution License 4.0

https://creativecommons.org/licenses/by/4.0/deed.en_US

Dr. Prashant Mani, Associate Professor, SRMIST, NCR Campus, Ghaziabad, Has completed B. Tech Electronics \& Instrumentation Engineering, M. Tech VLSI Design and Ph. D VLSI. He has published more than 40 papers in international journals and conferences.

Dr. Pankaj Singh, Assistant Professor, SRMIST, NCR Campus, Ghaziabad, Has completed B. Tech in Electronics Engineering, M. Tech and Ph. D ECE. He has published more than 33 papers in international journals and conferences.

Mr. Abhshek Singhal, Assistant Professor, SRMIST, NCR Campus, Ghaziabad, Has completed B. Tech in Electronics Engineering, M. Tech (Digital Signal Processing ). He has published several papers in international journals and conferences.

Mr. Apoorv Katiyar, Graduate Scholar, SRMIST, NCR Campus, Ghaziabad, Has completed B. Tech in Electronics Engineering. He has published more than 3 papers in international conference 